\title{
Role of Magnetic Resonance Imaging of Brain in the Evaluation of Paediatric Epilepsy
}

\author{
R. A. Umap ${ }^{1}$, Navid Shattari², Shephali Pawar ${ }^{3}$ \\ ${ }^{1}$ Professor, ${ }^{2}$ Senior Resident, ${ }^{3}$ Professor and Head, Department of Radiodiagnosis, BJGMC Pune, Maharashtra, India. \\ Corresponding author: Navid Shattari, Senior Resident, Department of Radiodiagnosis, BJGMC Pune, Maharashtra, India.
}

DOI: http://dx.doi.org/10.21276/ijcmsr.2020.5.1.3

BY-NC-ND

How to cite this article: R. A. Umap, Navid Shattari, Shephali Pawar. Role of magnetic resonance imaging of brain in the evaluation of paediatric epilepsy. International Journal of Contemporary Medicine Surgery and Radiology. 2020;5(1):A10-A15.

\section{A B S T R A C T}

Introduction: Epilepsy is a condition in which seizures are triggered recurrently from within. It is considered to be present when two or more unprovoked seizures occur at an interval greater than $24 \mathrm{hrs}$ apart. Magnetic Resonance Imaging (MRI) is the imaging modality of choice due to its ability to depict neuroanatomy, excellent grey white matter differentiation, status of myelination and detection of focal structural brain lesions. Study aimed to detect and characterize the lesions causing epilepsy and to detect frequency of etiological factors responsible for epilepsy in pediatric age group (0 - 12 years).

Material and methods: A total number of 100 patients who presented with symptoms suggestive of Epilepsy (EpilepsyTwo or more episodes of unprovoked seizures 24 hours apart) under 12 years were imaged with 1.5 Tesla GE-signa Hdxll MRI machine in the department of radiology over a period of 18 months. The data obtained was coded and entered into Microsoft Excel Worksheet. The categorical data was expressed as rates, ratios, proportions and percentages.

Results: In the age group of 0-12 years the most common etiology was Anoxia and Hypoxic ischeamic encephalopathy (31.5\%) followed by infection (25.8\%) and developmental malformations (13.5\%) respectively. Mesial temporal sclerosis, Phakomatosis, demyelinating diseases, neoplasm and vascular etiologies also plays significant role.

Conclusion: MRI plays a significant role in evaluation of pediatric patients presenting with epilepsy with proper seizure protocol to establish the correct diagnosis and plan the management according to diagnosis as well as helps in prognosis.

Keywords: Magnetic Resonance Imaging, Brain, Paediatric Epilepsy

\section{INTRODUCTION}

A seizure is defined as a sudden, paroxysmal electrical discharge from the central nervous system resulting in involuntary motor, sensory or autonomic disturbances with or without alteration in sensorium. The age and neurodevelopmental maturity status defines the clinical manifestation and type of seizure disorder. About $5 \%$ children are at risk of having a seizure and half of them encounter the first seizure in infancy. Prevalence is more in the neonatal period (almost $1 \%$ in term and 20\% in preterm). Epilepsy is a condition in which seizures are triggered recurrently from within. The collective life time incidence of epilepsy is $3 \%$, more than half the cases begin in childhood. The annual prevalence of epilepsy is lower (0.5-0.8\%) because many children exceed epilepsy. Electroencephalograph and neurosonogram are often the initial diagnostic workup for seizure activity. They have the benefits of being non-invasive and avoiding radiation exposure. Computed tomography is helpful in detection of calcific foci; however, it has the risk of radiation exposure. Magnetic Resonance Imaging (MRI) is the imaging modality of choice due to its ability to depict neuroanatomy, excellent grey white matter differentiation, status of myelination and detection of focal structural brain lesions.
Study aimed to detect and characterize the lesions causing epilepsy in pediatric age group $(0-12$ years $)$ and to detect frequency of etiological factors responsible for epilepsy in pediatric age group ( 0 - 12 years).

\section{MATERIAL AND METHODS}

Current hospital based prospective study was done on total number of 100 patients who presented with symptoms suggestive of Epilepsy under 12 years and were imaged with 1.5 Tesla GE-signa HdxII MRI machine in the department of radiology over a period of 18 months. The ethical clearance was obtained from Ethics Committee of B.J.government medical college, Pune. Patients fulfilling the selection criteria were informed about the purpose and nature of the study and were enrolled after obtaining a written informed consent.

Inclusion criterion: All pediatric patients (age under 12 years) referred from OPD \& IPD who presented with epilepsy.

Exclusion criteria: 1. Claustrophobic patients. 2. Patients with metallic implants considered contraindicated for MRI imaging. 3. Patients with trauma. 4. Patients with febrile seizure disorders.

Recommended sequences: Conventional MR imaging was 
performed by taking T1W (TE $8.0 \mathrm{~ms}$, TR $480 \mathrm{~ms}$ ), T2W (TE $102.9 \mathrm{~ms}$, TR $4780 \mathrm{~ms}$ ), and FLAIR (TE $92.2 \mathrm{~ms}$, TR $8002 \mathrm{~ms}$ ) sequences. Post gadolinium (dose $0.1 \mathrm{mmol} / \mathrm{kg}$ ) enhanced MRI was performed in Axial and Sagittal planes in selected cases depending on findings on non-contrast study or clinical suspicion. DWI (TE $83 \mathrm{~ms}$, TR $5025 \mathrm{~ms}$ ) and GRE (Gradient recalled echo) axial performed in all cases. When required, MR spectroscopy, MR venography and MR angiography including TOF was done.

\section{STATISTICAL ANALYSIS}

The data obtained was coded and entered into Microsoft Excel Worksheet. The categorical data was expressed as rates, ratios, proportions and percentages.

\section{RESULTS}

This was a prospective study of 18 months duration, done at department of Radiodiagnosis, B.J. government medical college, Pune evaluating the role of Magnetic resonance imaging of brain in the evaluation of paediatric epilepsy. This study includes MRI brain evaluation of 100 cases of pediatric patients aged 0-12 years who presented with epilepsy.

Maximum patients were in the age group 0-3 years (60.0\%). Males (65.0\%) were affected more commonly than females (35.0\%). Generalized seizures (68.0\%) were more prevalent than focal seizures (21.0\%). Majority of the patients showed abnormal MRI findings (89.0\%). The most common etiology for epilepsy was Hypoxic ischemic encephalopathy (31.5\%) followed by Infection (25.8\%) and Developmental malformations (13.5\%) (fig-1). According to age

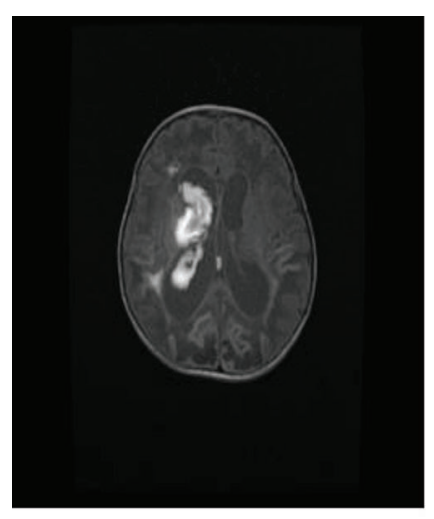

AXIAL TIW

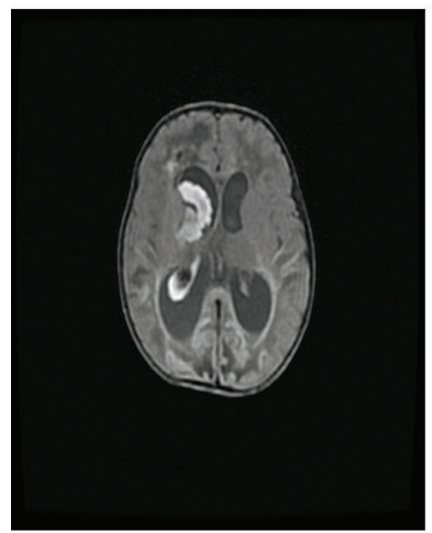

AXIAL FLAIR

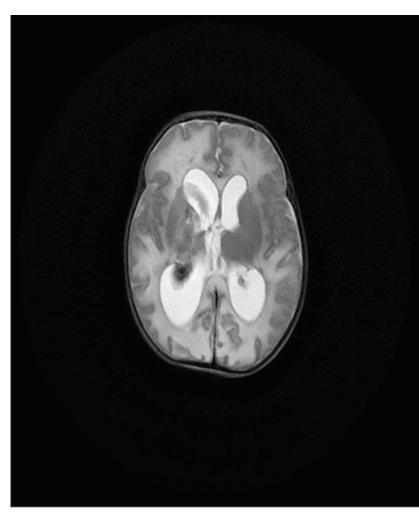

AXIAL T2W

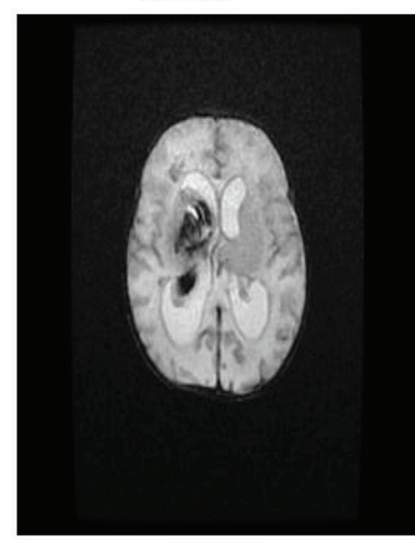

AXIAL GRE
Case-1: Hypoxic ischaemic encephalopathy with germinal matrix haemorrhage distribution, anoxia and hypoxic ischemic encephalopathy (45.2\%) was the most common cause in 0-3 years, followed
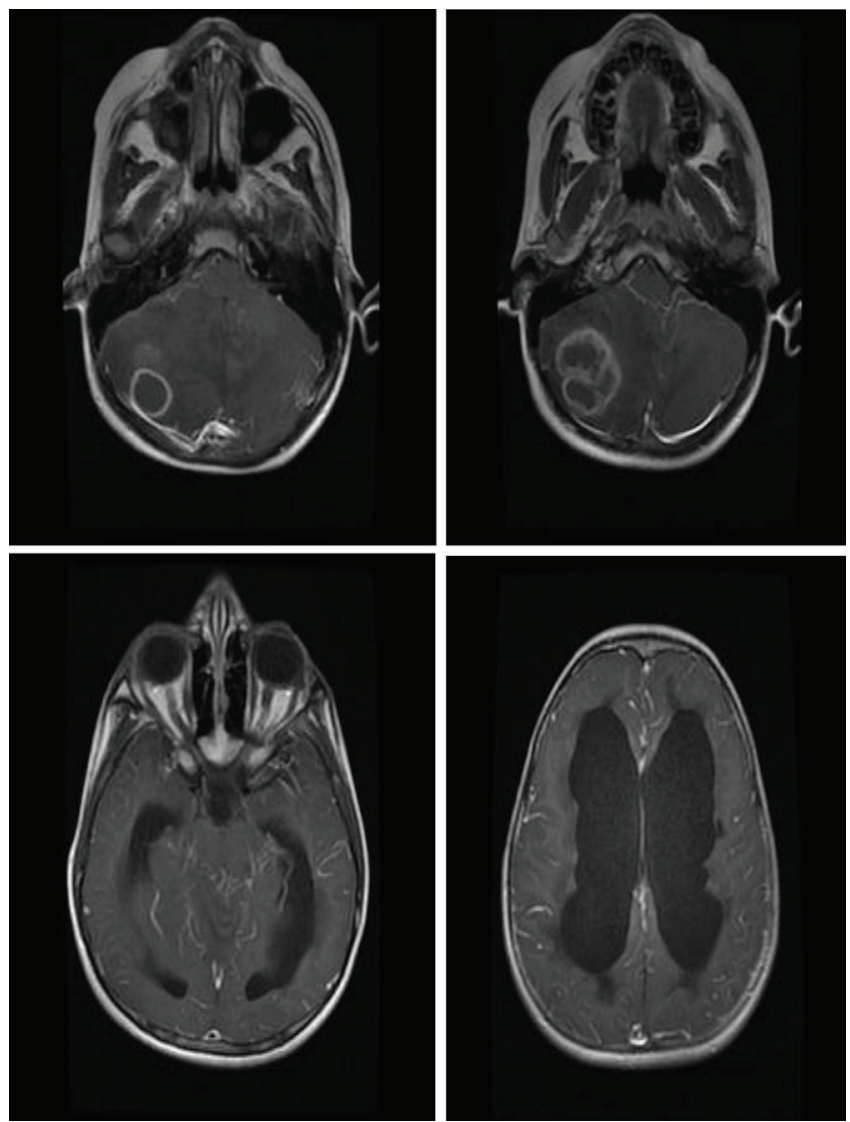

Case-2: Giant tuberculoma with obstructive hydrocephalus

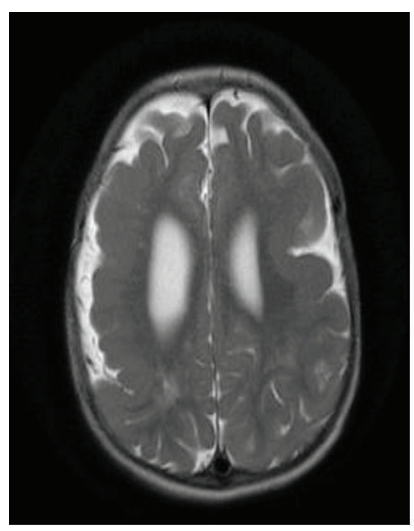

AXIAL T2W

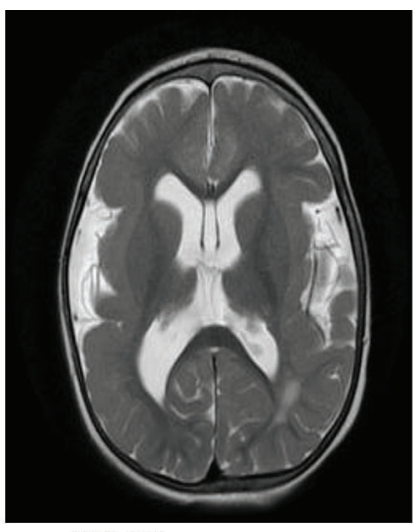

AXIAL T2W

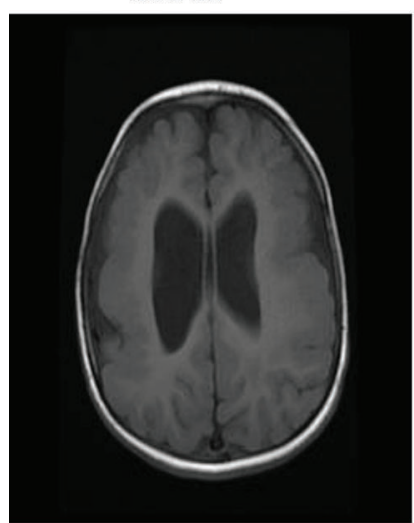

AXIAL T1W

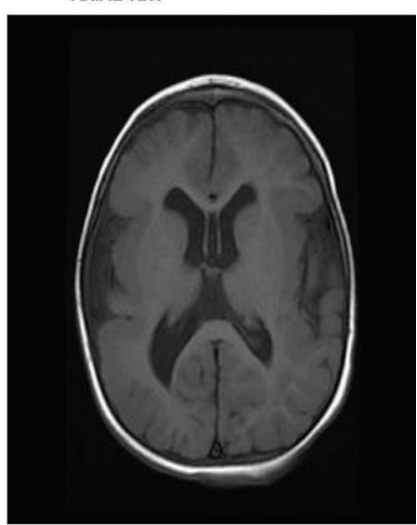

AXIAL T1W

Case-3: Focal cortical dysplasia with pachygyria 


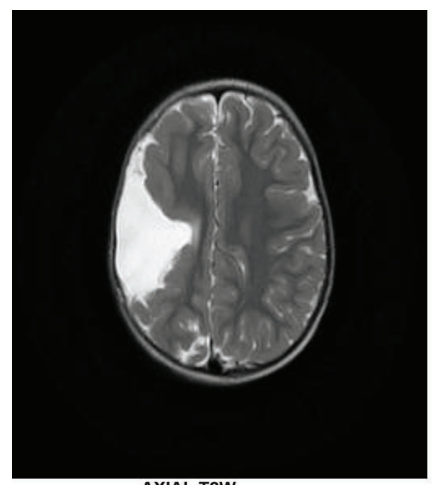

AXIAL T2W

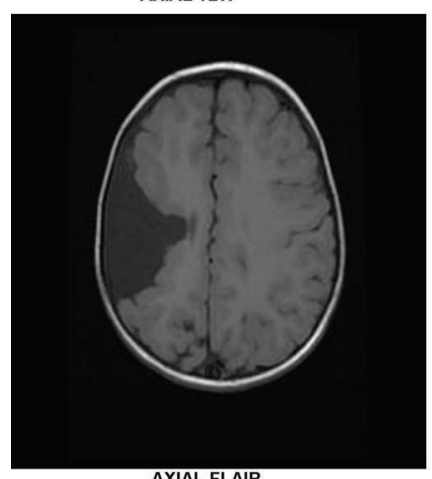

AXIAL FLAIR

Case-4: Schizencephaly

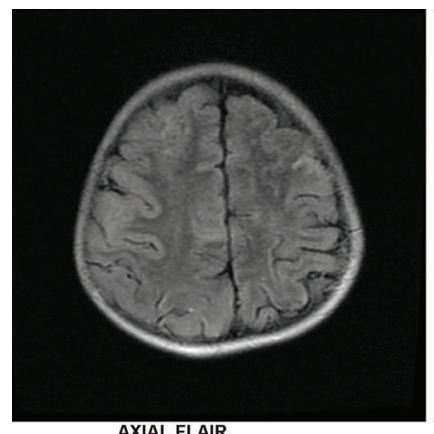

AXIAL FLAIR

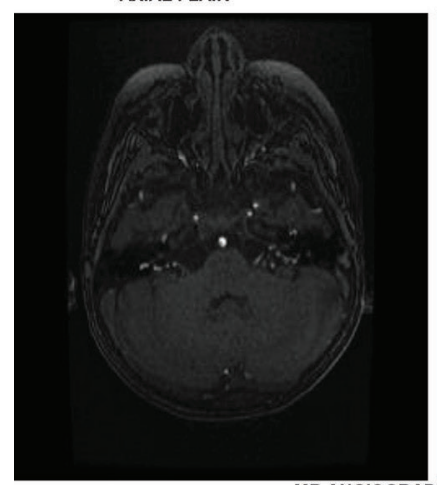

MR ANGIOGRAPHY (3D TOF - 2SLAB)

Case-5: Moyamoya disease

by infection (18.8\%) and developmental malformations (13.2\%). In all other age groups, infection was the most common etiology. Mesial temporal sclerosis (71.4\%) was the most common cause implicated in isolated temporal lobe epilepsy. Hippocampal atrophy (100\%) and secondary changes (dilatation of temporal horn) (100\%) were most frequent neuroimaging findings in mesial temporal sclerosis being present in all patients of that category. Most common pathology in developmental malformations was focal cortical

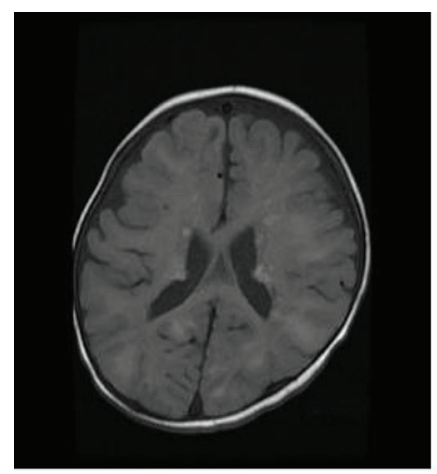

AXIAL T1W

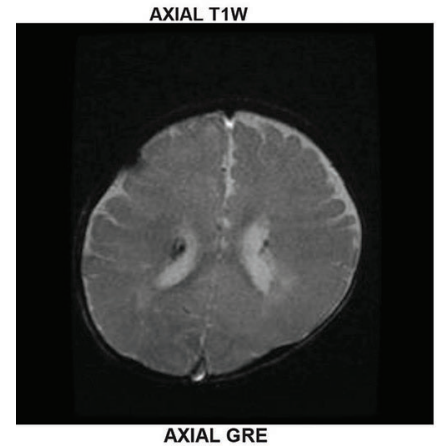

Case-6: Tuberous sclerosis

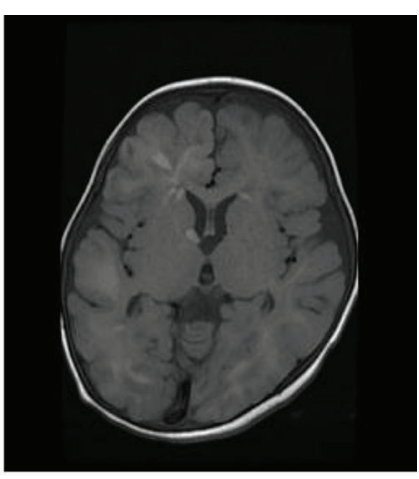

AXIAL T1W

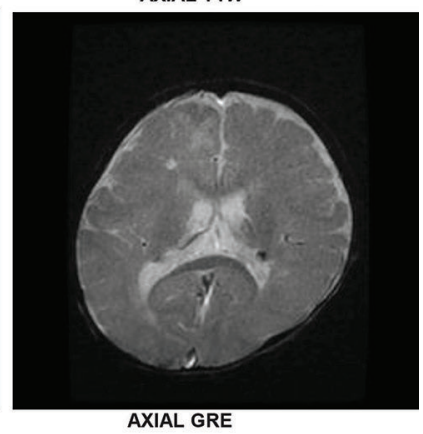

dysplasia (33.3\%). Most common pathology in phakomatoses was Tuberous sclerosis (60.0\%). Most common neuroimaging finding in patients of anoxia and Hypoxic ischemic encephalopathy was Leukomalacia (60.7\%). Most common infection in our study was Tuberculosis (30.4\%) followed by Neurocysticercosis (26.0\%). Most common neuroimaging finding in Tuberculosis was Tuberculoma (71.4\%). Most common pathology in patients with vascular etiology was arterial infarct (excluding tuberculous and including moya moya disease) (66.7\%). Neoplasms and demyelinating pathologies constituted a small percentage of the sample (4.5\%). Least common etiology in our study was inherited metabolic disorders (1.1\%).

Areas of symmetric confluent hyperintensities noted involving subcortical and periventricular white matter of bilateral frontoparietal region with areas of blooming involving right germinal matrix extending into intraventricular region (case-1). Post contrast Axial T1W images showed large well defined peripherally enhancing lesion noted in right half of cerebellum associated with perilesional edema causing compression of fourth ventricle resulting into hydrocephalus (case-2). Cortical thickening is noted involving bilateral cerebral parenchyma along with loss of grey white matter differentiation (right $>$ left). The gyral pattern is simplified with areas of pachygyria is noted (case-3). Fairly defined CSF intensity cystic lesion forming cleft noted involving right frontoparietal region and lined by grey matter which is seen extending from cortex upto right lateral ventricle (case-4). Gyriform thickening with FLAIR hyperintense signal noted along bilateral frontoparietal region with areas of restricted diffusion on DWI. MR Angiography showing narrowing of bilateral ICA with severe narrowing of supraclinoid portion of bilateral ICA. Multiple pial collaterals are noted along bilateral cerebellopontine angle (case-5). There are confluent and discrete areas of T1W hyperintensities noted involving 


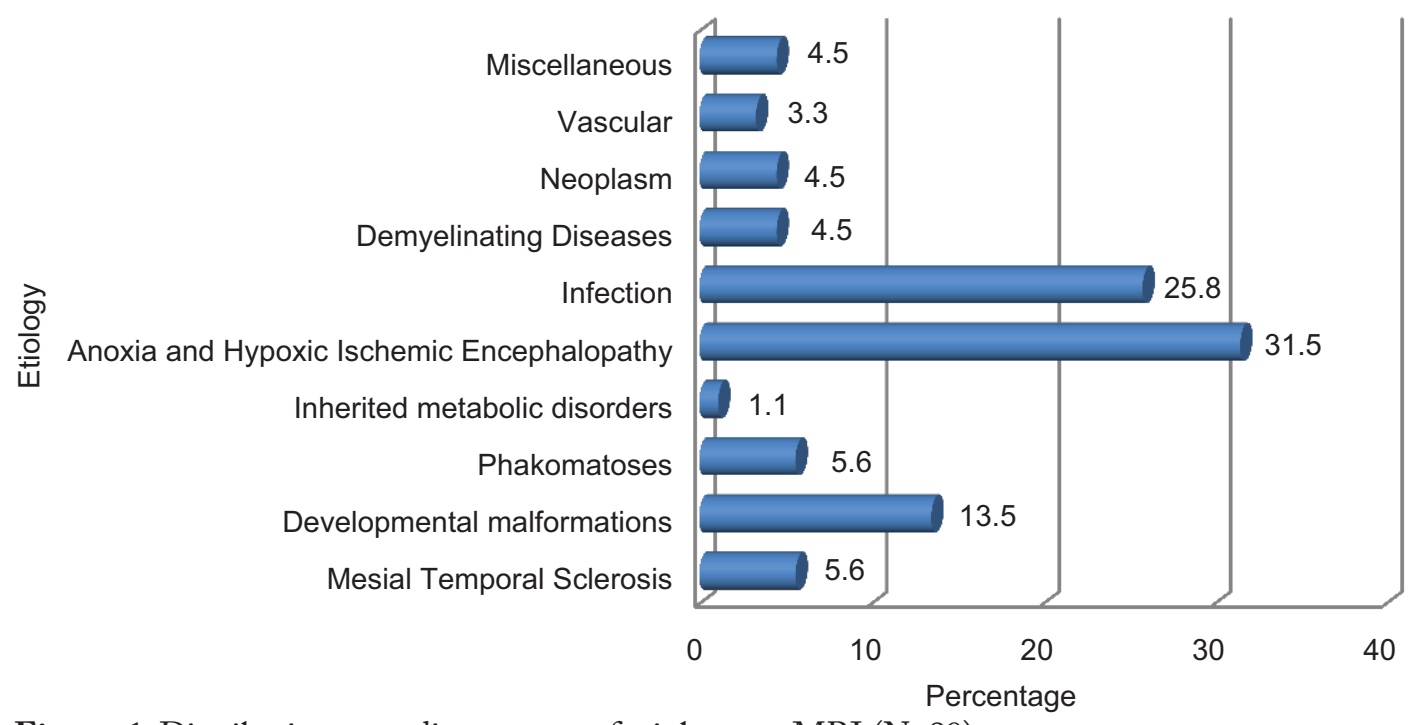

Figure-1: Distribution according to type of etiology on MRI (N=89)

subcortical and periventricular white matter of bilateral frontoparietal region. Few subependymal nodules are seen which shows corresponding blooming on GRE images s/o calcification (case-6).

\section{DISCUSSION}

In our study of total 100 patients, maximum 60 patients $(60.0 \%)$ were in the age group of $0-3$ years followed by $10-$ 12 years which included 20 patients $(20.0 \%)$. Least number of patients were 8 (8.0\%), in the age group of 7-9 years. Our study is in discordance with the study done by Gulati $\mathrm{P}$ et $\mathrm{al}^{1}$ in which maximum patients were in the age group 6-12 years.

In our study of 100 patients, $65.0 \%$ were males and 35.0\% were females. Male:Female ratio was 1.8:1. Our study correlates with the study done by Sanghvi JP et al ${ }^{2}$ in which $60.5 \%$ were males and $31.7 \%$ were females. In our study of 100 patients, maximum number of patients, $68.0 \%$ presented with generalized seizures. Our study correlates with the study done by Rasool A et $\mathrm{al}^{3}$ in which Generalized seizures constituted the major seizure group being present in as many as $42 \%$ of patients. In this study of total 100 patients, 89 patients (89.0\%) had abnormal MRI findings. Our study is comparable with the study done by Kuzniecky $\mathrm{R}$ et $\mathrm{al}^{4}$ in which MRI revealed abnormalities in $84 \%$ of patients. In our study, $31.5 \%$ patients have hypoxic ischemic encephalopathy (HIE) followed by Infection which was seen $25.8 \%$ of patients. Developmental Malformation was seen in $13.5 \%$ of patients. Phakomatosis and Mesial temporal sclerosis causes constituted 5 patients each (5.6\%). Demyelinating diseases, Neoplasm and Miscellaneous causes constituted 4 patients each (4.8\%). Vascular cause was seen in 3 patients (3.3\%). Least common was inherited metabolic disorders comprising of 1 patient (1.1\%). Our study does not correlate with Gulati P, Jena A.N. et $\mathrm{a}^{5}$ study, in which infection as the major etiology in 53.3\%, followed by neoplastic in $17.3 \%$, cerebrovascular accident in $6.6 \%$ and miscellaneous causes in rest 22.6\%. Our study does not correlate with Parihar Ravi Kumar et $\mathrm{al}^{6}$ study, in which neurocysticercosis (NCC) was seen in 55.81\%, tuberculoma in 29.91\%, normal MRI findings in $8.30 \%$ and findings suggestive of cerebritis, sturge weber syndrome and porencephalic cyst in 6.98\%. Our study does not correlate with Chaurasia $\mathrm{R}$ et $\mathrm{al}^{7}$ study, in which most common etiology of epilepsy was CNS Tuberculosis $30.3 \%$, followed by Neurocysticercosis $11.0 \%$ and encephalitis $7.9 \%$. In our study, 7 out of 89 patients $(7.8 \%)$ had isolated temporal lobe involvement. Mesial temporal sclerosis was most commonly seen in $71.4 \%$, followed by Focal cortical dysplasia and Tuberculosis with tuberculoma in 14.3\% patient each. Our study correlates with study done by Sales $\mathrm{LV}$ et $\mathrm{al}^{8}$, in which out of 31 patients with temporal lobe epilepsy, mesial temporal sclerosis was seen in 9 (29.0\%), dysplasia in $8(25.8 \%)$, tumors in $2(6.4 \%)$, arachnoid cyst in $1(3.2 \%)$ and choroid cyst in 1 (3.2\%). In our study, out of 4 patients with mesial temporal sclerosis, hippocampal atrophy and secondary change (dilatation of temporal horn) was found in $100 \%$ of patients. Loss of hippocampal architecture was seen in $75 \%$ and hippocampal T2, FLAIR hyperintensity in $66.7 \%$ of patients. Our study is in discordance with $\mathrm{Ng}$ YT et a $1^{9}$ study, in which out of 24 patients with mesial temporal sclerosis, hippocampal T2 hyperintensity was seen in 20 patients (83.3\%), Hippocampal atrophy in 19 patients (79.2\%), loss of hippocampal architecture in 13 patients (54.2\%) and secondary change (decreased hippocampal T1weighted signal) in 3 patients (12.5\%). In our study, 12 patients had malformations of development. Focal cortical dysplasia was the most common in 4 patients (33.3\%). Corpus callosal dysgenesis/agenesis and polymicrogyria constituted 3 patients (25.0\%) each. Pachygyria constituted 2 patients (16.7\%) while Microcephaly with simplified gyral pattern, Heterotopia, Hemimegalencepahly and Holoprosencephaly (Lobar) constituted the remainder with 1 patient (8.3\%) in each category. 4 patients (33.3\%) had multiple pathologies. Our study correlates with study done by Mittal GK et $\mathrm{al}^{10}$, in which out of 54 patients with Malformations of cortical development, Focal cortical dysplasia was the most common seen in 16 patients (29.6\%), followed by Schizencephaly in 8 (14.8\%), Polymicrogyria in $8(14.8 \%)$, DNET in 6 (11.1\%), Lissencephaly in $5(9.3 \%)$, Ganglioglioma in 3 (5.6\%), Heterotopia in $3(5.6 \%)$, 
Hemimegalencephaly in $2(3.7 \%)$, cortical hamartomas of tuberous sclerosis in $2(3.7 \%)$ and mixed lesion in 1 (1.8\%). Our study had 5 patients of Phakomatoses, 3 (60.0\%) had Tuberous sclerosis, Sturge weber syndrome in 1 (20.0\%) and Neurofibromatosis in 1 (20.0\%). Our study does not correlate with study done by Dietrich $\mathrm{RB}$ et $\mathrm{al}^{11}$, in which neuroimaging study of 29 patients with medically intractable seizures was done. The study included other studies than MRI for neuroimaging and Neurophakomatoses was the etiology in 5 $(17.4 \%)$ patients. In the study, most common phakomatoses was Sturge weber syndrome seen in 3 patients $(60.0 \%)$, Tuberous sclerosis in $1(20.0 \%)$ and Neurofibromatosis in 1(20.0\%). In our study, anoxia and HIE was seen in 28 patients. 16 patients $(57.2 \%)$ were Preterm and 12 patients (42.8\%) were term. Among the neuroimaging findings, most common was Leukomalacia was seen in 17 patients $(60.7 \%)$, followed by Cystic changes in 12 patients (42.8\%), White matter volume loss in 9 patients $(32.1 \%)$ and Intracranial haemorrhage in 1 patient (3.5\%). Our study does not correlate with study done by Alam $\mathrm{A}$ et $\mathrm{al}^{12}$, in which 45 children with history of perinatal asphyxia were studied with MRI. Most common finding in this study was haemorrhage seen in 16 patients (35\%) followed by periventricular leukomalacia in 13 patients $(28.8 \%)$. This could be due to maximum cases of haemorrhage being diagnosed on ultrasound screening in patients with history of perinatal asphyxia in our department and thus less referral for MRI. In our study, Infectious etiology was seen in 23 patients in which most common was tuberculosis found in 7 patients (30.4\%), followed by neurocysticercosis in 6 patients $(26 \%)$, encephalitis in 3 patients (13.0\%), Meningoencephalitis and Pyogenic abscess in 2 patients (8.7\%). Meningitis (excluding tuberculous), Subdural empyema and TORCH infection constituted 1 patient (4.3\%) each. In Gulati P, Jena A.N. et $\mathrm{al}^{5}$ study, out of 345 patients with abnormal MRI, tuberculoma was the most common etiology and was seen in 98 (28.4\%), followed by neurocysticercosis in $86(24.9 \%)$. In Chaurasia R et $\mathrm{al}^{7}$ study, most common cause of epilepsy was CNS tuberculosis (30.3\%), followed by Neurocysticercosis (11.0\%) and Encephalitis (7.9\%). Our study thus correlates with the above mentioned studies. In our study, Tuberculosis was found in 7 patients. Out of them, tuberculomas were seen in $71.4 \%$, followed by leptomeningeal enhancement in $57.1 \%$, infarcts in $28.6 \%$, tuberculous abscess in $14.3 \%$ and communicating hydrocephalus in $14.3 \%$. Our study is in concordance with Chaurasia $\mathrm{R}$ et $\mathrm{al}^{7}$ study, in which out of 191 abnormal MRI, CNS tuberculosis was seen in 58 patients (30.3\%). Of them, most common neuroimaging finding was tuberculoma seen in 30 patients (15.7\%) followed by leptomeningeal enhancement in 28 patients (14.6\%). Out of total 89 abnormal MRI, neoplasm was seen in $4(4.5 \%)$ patients. Ependymoma was seen in 2 patients (50\%). Medulloblastoma and Pontine glioma was seen in 1 patient (25\%) each. Our study is not in concordance with Zajac A et $\mathrm{al}^{13}$ study, in which out of total 45 children studied, only 2 patient (4.4\%) had brain tumors. One patient had DNET and another patient had hypothalamic hamartoma. In our study, neuroimaging findings were suggestive of vascular cause in 3 patients. $2(66.7 \%)$ of them had arterial infarcts (excluding tuberculous and including moya moya disease), 1 (33.3\%) had venous infarct. Our study does not correlate with the study done by Wongladarom $\mathrm{S}$ et $\mathrm{al}^{14}$, in which vascular disorder was responsible for epilepsy in 5 patients (5\%). Out of them, 2 patients (40\%) had Moya moya disease, 1 patient (20\%) had cavernous angioma, 1 patient (20\%) had arteriovenous malformation and the remaining 1 patient (20\%) had bilateral carotid occlusion. Out of total 100 patients included in our study, 89 patients had abnormal MRI. Out of total 53 patients in the age group of 0-3 years, Anoxia and HIE was the most common etiology in seen in 24 patients (45.2\%) followed by Infection in 10 patients (18.8\%). Developmental malformations were also most common in this age group and were found in 7 patients (13.2\%). Out of total 12 patients in age group 4-6 years, 8 patients in age group 7-9 years and 16 patients in age group 10-12 years, most common etiology was Infection and was seen in 3 patients (25.0\%), 3 patients (37.5.\%) and 7 patients (43.7\%) respectively. For etiology in age group 0-3 years, our study correlates well with Khreisat WH. ${ }^{15}$ study, in which children suffering from epilepsy below the age of 2 years were studied. The most common etiological factor found in this study was perinatal asphyxia seen in 55\%, followed by CNS infection in 15\%, anomalies of central nervous system in (9\%), head injuries in (8\%), congenital and heredofamilial disorders in (8\%) and prematurity in (5\%). For etiology in older age group, our study correlates with Parihar Ravi Kumar et $\mathrm{al}^{6}$ study, in which children in the age group of 28days to 18 years with partial seizures were studied. 6 patients $(66.6 \%)$ in age group of 28 days-5years, 18 patients $(85.7 \%)$ in age group of $>5-10$ years and 12 patients $(92.3 \%)$ in age group $>10-18$ years had infection as the most common etiology. Thus infection had major burden in causing epilepsy with increasing age group. This study had 4 patients with abnormal MRI grouped under miscellaneous cause. Post ictal edema was seen in 1 patient (25.0\%), Aqueductal stenosis in 1 patient (25.0\%), Arachnoid cyst in 1 patient (25.0\%) and Idiopathic intracranial hypertension in 1 patient (25.0\%). We found 4 patients showing imaging findings of demyelination in our study. One of the patient (25\%) had classical presentation of ADEM. MRI findings of patchy hyperintense signal in bilateral corona radiate and central semiovale on T2W and FLAIR images, showing restricted diffusion with nodular enhancement on post contrast T1W images. No comparable studies were available as some of the published studies which we have gone through had discussed the above etiologies under a single heading.

\section{CONCLUSION}

MRI plays an invaluable role in the evaluation of pediatric patients with seizure disorder. Accurate diagnosis of cause of seizure is important for treatment decision. With its high spatial resolution, excellent inherent soft tissue contrast, multiplanar imaging capability and lack of ionizing radiation; MRI has emerged as a versatile tool in imaging of pediatric patients with seizures. MRI not only identifies specific epileptogenic substrates, but also helps in determining specific treatment and predicts prognosis. Employing appropriate imaging protocols and reviewing the images in systemic manner 
helps in the identification of subtle epileptogenic structural abnormalities. MR imaging is superior neuroimaging with no radiation exposure and could be the first investigation of choice in epileptic syndrome, developmental cortical malformations and mesial temporal sclerosis. Its ability in identifying subtle lesions, location and extent of the lesions is excellent. MRI helps in evaluation of patients presenting with refractory seizures undiagnosed by other imaging modalities and thus helps to prevent further unnecessary investigations. MRI plays an important role in evaluation of children with newly diagnosed epilepsy especially those with abnormal neurological examination, focal seizures or focal EEG abnormalities. Hence we conclude that MRI plays a significant role in evaluation of pediatric patients presenting with epilepsy and it is the first imaging modality of choice with proper MRI seizure protocol to establish the correct diagnosis, plan the management according to diagnosis and helps in prognosis.

\section{REFERENCES}

1. Gulati P, A Jena, Tripathi RP, Gupta AK, Magnetic resonance imaging in childhood epilepsy. Indian pediatr. 1991; 28(7):761-5.

2. Sanghavi JP, Rajadhyaksha SB, Uresekar M. Spectrum of congenital CNS malformation in paediatric epilepsy. Indian Pediatr 2004; 41(3):831-8.

3. Rasool A, Choch SA, Wani NA, Ahmad SM, Iqbal Q. Role of EEG and neuroimaging in first onset afebrile and complex febrile seizures in children from Kashmir. Journal of paediatric Neurosciences. 2012; 7(1):9-15.

4. Kuzniecky R, Murro A, King D. Magnetic resonance imaging in childhood intractable partial epilepsies: pathological correlations 1993;43(4):681-687.

5. P. Gulati, A.N. Jena, V. Puri, P.C. Sanchetee. MRI (magnetic resonance imaging) spectrum of epilepsy. J. Indian Med. Assoc., 1994;92(6):110-112.

6. Parihar RV, Gupta AK, Saini G, Dev G. Role of MRI imaging of brain in paediatric patient with partial epilepsies. JK Sci. 2012;14(1):60-4.

7. Chaurasia R, Singh S, Mahur S, Sachan P. Imaging in paediatric epilepsy, Spectrum of abnormalities detected on MRI. J Evolv Med Dent Sci 2013;19(3):3377-87.

8. Sales LV, Velasco TR, Relative frequency, clinical, neuroimaging, and postsurgical features of pediatric temporal lobe epilepsy. 2006; 39(10):1365-72.

9. $\mathrm{Ng}$ YT, McGregor AL, Duane DC, Jahnke HK, Bird CR, Wheless JW. Childhood mesial temporal sclerosis. J Child Neurol 2006; 21(6):512-7.

10. Mittal GK, Ganguly G, Bhattacharyya KB, Pandit A, Biswas A, Roy A, et al. Epilepsy patients with malformations of cortical development: Experience from a tertiary care centre in Eastern India. J Pediatr Neurol 2014; 12(2):117-26.

11. Dietrich RB, El Saden S, Chugani HT, Bentson J, Peacock WJ. Resective surgery for intractable epilepsy in children: Radiologic evaluation. Am J Neuroradiol 1991;12(4):1149-58.

12. Alam A, Sahu S. Magnetic resonance imaging in evaluation of periventricular leukomalacia. Med J Armed Forces India 2010;66(1):374-80.

13. Zajac A1, Herman - Sucharska I, Kroczka S, Kubik
A, Nardezewska-Szcepanik M. Brain MRI data in children with so called primary generalized seizures. PrazegilLek. 2007; 64(11):942-5.

14. Wongladarom S, Laothamatas J, Visudtibhan A, Sawatsut P. Magnetic resonance imaging in epileptic paediatric patient. Review of experience of ramathibodi hospital. J Med Assoc Thai 2004;87(1):1092-9.

15. Khreisat WH. Clinical profile of epilepsy during the first two years of life. Pak J Med Sci 2006; 22(5):55.

\section{Source of Support: Nil; Conflict of Interest: None}

Submitted: 22-10-2019; Accepted: 25-11-2019; Published online: 17-01-2020 